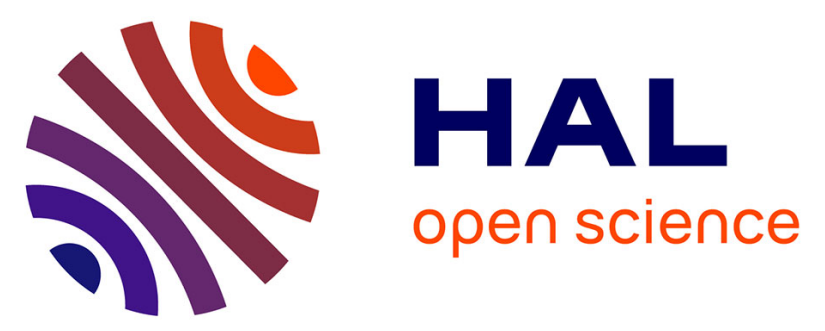

\title{
Identification of Eggplant Young Seedlings Infected by Root Knot Nematodes Using Near Infrared Spectroscopy
}

\author{
Wei Ma, Xiu Wang, Lijun Qi, Dongyan Zhang
}

\section{To cite this version:}

Wei Ma, Xiu Wang, Lijun Qi, Dongyan Zhang. Identification of Eggplant Young Seedlings Infected by Root Knot Nematodes Using Near Infrared Spectroscopy. 10th International Conference on Computer and Computing Technologies in Agriculture (CCTA), Oct 2016, Dongying, China. pp.93-100, 10.1007/978-3-030-06155-5_9. hal-02180004

\section{HAL Id: hal-02180004 \\ https://hal.inria.fr/hal-02180004}

Submitted on 12 Jul 2019

HAL is a multi-disciplinary open access archive for the deposit and dissemination of scientific research documents, whether they are published or not. The documents may come from teaching and research institutions in France or abroad, or from public or private research centers.
L'archive ouverte pluridisciplinaire HAL, est destinée au dépôt et à la diffusion de documents scientifiques de niveau recherche, publiés ou non, émanant des établissements d'enseignement et de recherche français ou étrangers, des laboratoires publics ou privés. 


\title{
Identification of eggplant young seedlings infected by root knot nematodes using near infrared spectroscopy
}

\author{
Wei Ma ${ }^{1,2}$, Xiu Wang ${ }^{1,2(\bowtie)}$, Lijun $\mathrm{Qi}^{3}$, and Dongyan Zhang ${ }^{4}$ \\ ${ }^{1}$ Beijing Research Center of Intelligent Equipment for Agriculture, Beijing 100097, China \\ (Tel:86-010-51503346;e-mail:maw516@163.com;xiuwang@263.net). \\ ${ }^{2}$ National Engineering Research Center of Intelligent Equipment for Agriculture, Beijing 100097 \\ China(e-mail: xiuwang@263.net) \\ ${ }^{3}$ College of Engineering, China Agricultural University, Beijing 100083, China(e-mail: qilijun@cau.edu.cn) \\ ${ }^{4}$ School of Electronics and Information Engineering, Anhui University, Hefei 230601(zhangdy@ahu. edu. cn)
}

\begin{abstract}
In this paper, eggplant young seedlings infected by root knot nematodes were identified using near infrared spectroscopy. The main research on MSC and SG pretreatment method and PCA principal component extraction method with the combination of effects on model for classification. Results show:The best classification process is to do the first MSC after SG smoothing pretreatment, after using PCA extracted as the main component of the SIMCA input variables for classification, and achieved a classification average accuracy higher than $90 \%$. It is an effective method to classify the degree of infection of the root knot nematodes by using the visible and near infrared spectral characteristics of the eggplant leaves.
\end{abstract}

Keywords: Near-infrared spectroscopy, Soil disease, Disease identification

\section{INTRODUCTION}

The Facility agriculture is an important part of modern agriculture in China, and it plays an important role to stable supply of vegetables and fruit for the city. Eggplant is grown in many continuous years in agricultural production base in Beijing. The soil borne disease exacerbation has been caused by this practice, so the quality of fruit and vegetable decreased. In addition to the environment under high temperature and high humidity in greenhouse, it is more conducive to the growth of root knot nematodes in soil after continuous cropping.

Root knot nematode is known as "plant cancer". Root knot nematodes (Meloidogyne Incognita ) in the soil after the disease will reduce $10 \%-20 \%$ yield of the crop, serious up to $75 \%$ or more. The nematode is not only harmful to the plant itself, The wounds caused by the invasion is in favor of other pathogens such as bacteria and fungi, and other pathogens interaction. Agricultural production is particularly serious harm, especially the economic value of the higher crops such as tomato, gourd, etc. . Economic losses caused by nematode damage every year to reach more than 3 billion yuan in China. In recent years, with the rapid development of large area of solar greenhouse in China, the occurrence of root knot nematode disease in the area has continued to expand. Root knot nematode is a major disease for agricultural production in China[4], especially the first major disease in the protection vegetable production in greenhouse.

Early detection of vegetable root knot nematode disease has been a problem, because the root knot nematodes occurred in the underground. The disease is difficult to find in the daily management, it is too late to prevent and control when the symptoms of the disease performances in the part of the eggplant on the ground. And the disease can be spread with irrigation water, so it is difficult to eradicate once it occurs. On the other hand, due to perennial root knot nematode parasites in the soil and plant roots organization, the occurrence of this disease is often detected. Therefore, rapid detection for the disease has become one of the key problems in the research and production of agriculture. Now it is still a weak link for the prevention of root knot nematode and forecasting. Main methods include excess soil fumigation, mixed and irrigation root with the drugs before planting or uprooting. These methods is easy to 
bring secondary pollution for the soil and has the existing problem of low efficiency.

There are many disadvantages of detection pests method existing in the actual agricultural production. For example, there are many objective problems that the subjective influence, not quantitative and time lag using the artificial observation or experience to judge. In previous studies, there are count and chemistry methods of acquisition and analysis of root sample. This method is time-consuming, large loss and long cycle and the recognition accuracy and efficiency is not high. It is not easy to root disease sampling survey. Because the existing detection method of vegetable root knot nematode cannot achieve rapid, accurate and nondestructive on-line early detection, therefore the application of new technology and methods for early detection of disease are required.

Visible and near infrared spectroscopy has many characteristics, which is fast, does not destroy the sample, the stability is good, the efficiency high, has been widely used in many fields. Many near infrared spectroscopy analysis methods are classified as standard methods. The analysis object of facility agriculture crops from the original organizational structure and seed is extended to include plant roots, stems, leaves, the whole plant, fruits and so on, analysis from powder samples were extended to the analysis of complete grain samples, sample liquid, vegetables live samples. Vegetable production is mainly used for the prediction of disease, the harvest period forecast, the lack of plant nutrition elements, the fruit quality of non - destructive testing. Disease recognition has achieved some results based on the spectrum. Muir et al. studied the spectral reflectance of potato tuber when it does not appear lesion to achieve early detection. Because of the relatively unique chemical composition in the epidermis and tissues of different insect pests, the energy levels and rotational energy levels of the insect body have been produced by the absorption of near infrared energy. Therefore, the quantitative identification of insect pests can be carried out according to the difference of the absorption and reflection of the near infrared light from different insect pests. Chinese cabbage inoculated with clubroot were carried out early rapid detection by the application of Fourier transform infrared spectroscopy. This is groundbreaking for the detection of root diseases in the application of infrared spectroscopy. Research results show that the infected root samples in the infrared spectrum has a strong absorption peak, and healthy roots samples spectra without the peak. The clubroot of Chinese cabbage was detected fast in the early stage of latency using peak area variation of absorption peak. At the same time, the polymerase chain reaction (PCR) was used to detect the evidence. This study is of great help to the study of root diseases. The changes of chlorophyll fluorescence parameters of eggplant leaves were studied during the infection of root knot nematode, using the method of artificial inoculation for potted Eggplant Seedlings. Conclusion shows the infection of root knot nematodes had significant effect on the chlorophyll fluorescence characteristics of eggplant leaves.

In this study, we use principal component analysis to extract a small amount of characteristic wavelength variables. Based on the independent soft mode classification of cluster, the identification of the root knot nematodes with very representative root knot nematode was identified. At the same time to ensure the accuracy of pest identification, improve the recognition efficiency.

\section{$2.1 \quad$ Test Material}

Eggplant seeds were used as the Jingqie 13 from Vegetable Research Center, Beijing Academy of agricultural and forestry sciences. In August 2015 10, the control group of eggplant planted in greenhouse in plant protection and Environmental Protection Research Institute, Beijing Academy of agriculture and forestry. A total of 40 pots of eggplant seedlings. There are four control group, each group of 10 pots of eggplant. Nematode of test accination all come from the Plant protection and environmental 
protection research institute. The test instrument is a portable spectrometer of American ASD company (Analytical Spectral Device, FieldSpec Pro)supporting the use of hand-held blade clip in order to improve the accuracy. Its spectral sampling interval: nm 350 1000 interval is 1.4 and 1000 2500 nm interval is $2 \mathrm{~nm}$. After calibration by RS3 standard software, the spectra were collected and recorded.

\section{$2.2 \quad$ Test method}

The eggplant in flower pot was inoculated with root knot nematode in three different groups at 3 o'clock in the afternoon of August 22, 2015. In the first group, the 10 pots of Eggplant were inoculated with a small amount of inoculation, and each pot was inoculated with one thousand root knot nematodes. Second groups of 10 pots of Eggplant in accordance with moderate inoculation, each pot is about two thousand root knot nematode. Third groups of 10, according to the multi inoculating basin, each pot is about five thousand. The fourth group was blank control group, and no inoculation treatment was done. Inoculation was injected directly into the roots of the soil by the way of surrounding the roots. In September 12, 2015 10:30-11:30, Spectral reflectance of 40 sets of four sets of samples were collected in greenhouse. Before each test, the spectrometer is calibrated with a white board, which is used to reduce the influence of spectral noise. According to the test situation of each test after the end of a group to re calibrate the white board. The spectrometer was set up to get the average of 10 sets of data. The upper and lower parts of each basin were collected 2 times, each time the 10 data were collected for an average to get a total of 80 sets of data.

\section{Results and Analysis}

The spectrum values of four groups of eggplant infected nematodes were obtained, as shown in Figure 1. It can be seen that the spectral values of the four groups can not be distinguished directly.

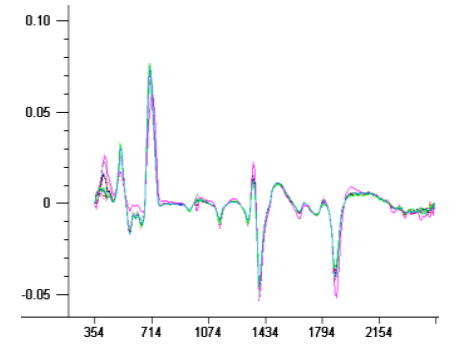

(a) 1000 nematode

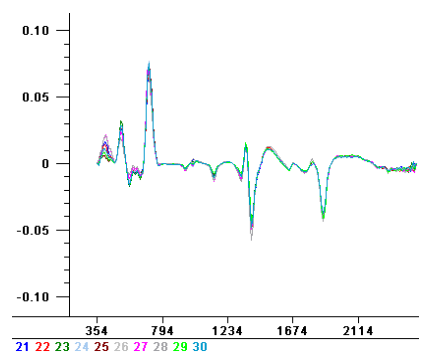

(c) 5000 nematode

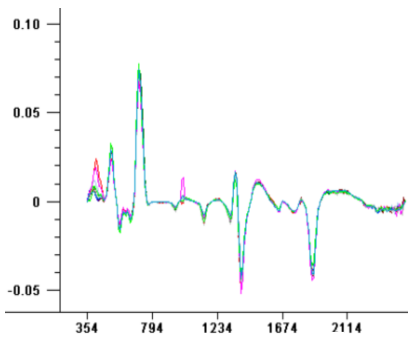

(b) 2000 nematode

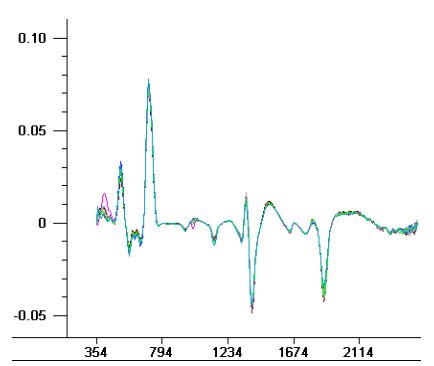

(b) Blank control

Fig. 1 The relationship between spectrum and quantity of inoculated nematodes

But there are differences in the visible and near infrared spectral region. By comparing the spectral values, we can find that the difference is not obvious. 


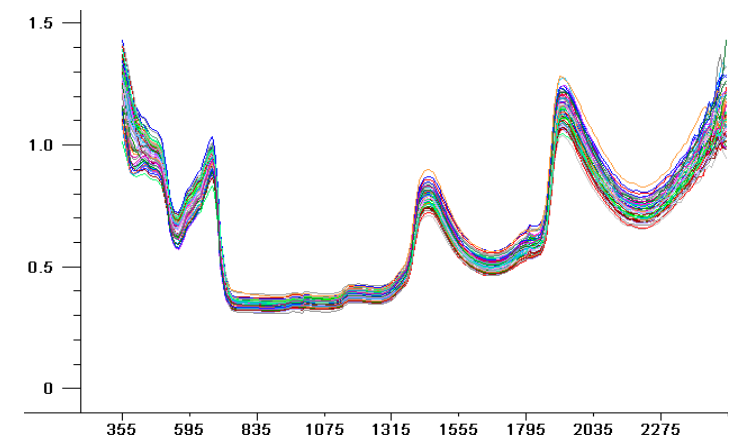

Fig. 2 Full spectrum of 4 groups

All spectra in Figure 2 there is some error due to Bring the light caused by blade clip leakage. The spectrum at the wavelength of $2000 \mathrm{~nm}$ above should be excluding because the error is too large.

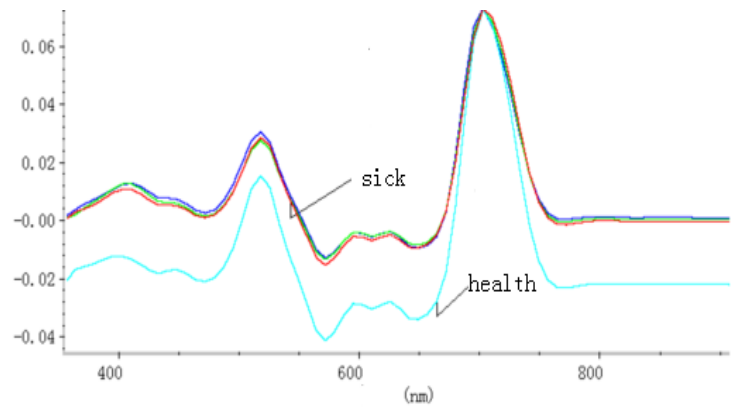

Fig. 3 Pre treated reflectance spectra

After smoothing the reflectance spectra of value to eliminate the error, according to the packet average from Figure 3 there are significantly differences distinguished the health eggplant and infected eggplant. In the range of 300 to $1000 \mathrm{~nm}$ there is a clear separation between the level of high and low.

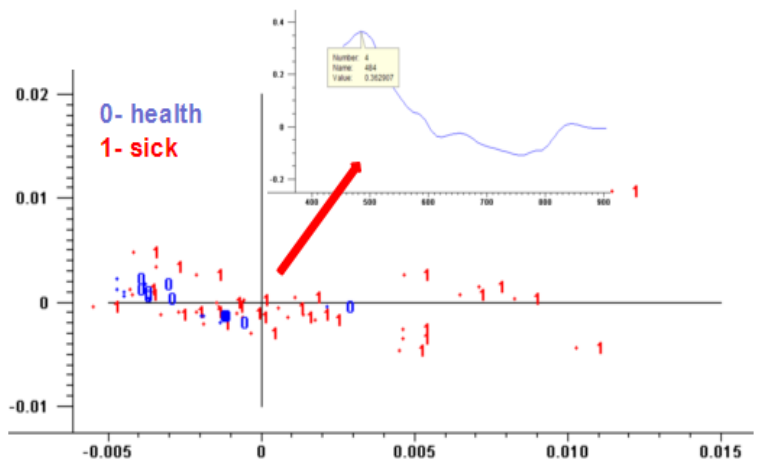

Fig. 4 Score chart of recognition model

In Figure 4, there are some overlapping of the samples in the identification of the grouping. In this study, the independent modeling of class analogy soft (SIMCA) classification method is applied to classify the eggplant in various control groups. SIMCA is a kind of recognition method based on principal component analysis. First on the modeling of the amount of each sample measured data matrix and the principal component analysis were used to establish each kind of principal component analysis mathematical model, and then calculate the unknown sample and modeling in the PCA model of distance, and by distance discriminant method to classify unknown samples. First, through repeated attempts to obtain the optimal sensitive 10 wavelengths(375 4755856757051305142517851805 1905), and then gradually reduce the number to 6 sensitive wavelengths(375 $475 \quad 675705 \quad 1425$ 1905) using principal component analysis. Fig 5 shows explained variance of the first 10 PCs of eggplant. 


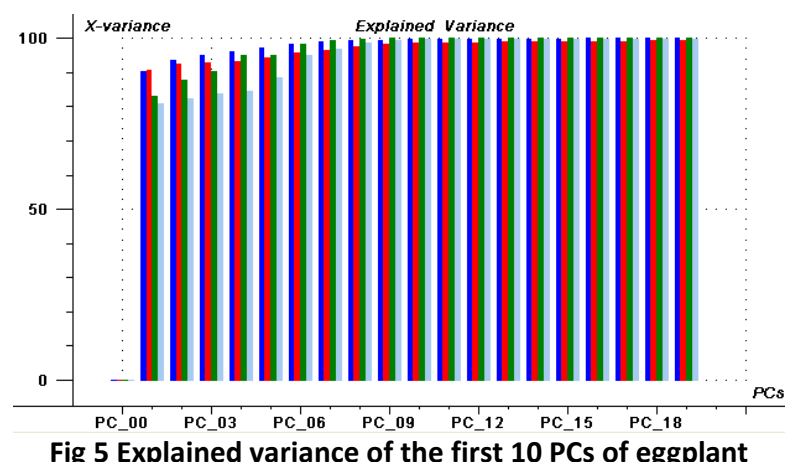

The model 1 uses the 1 order 3 order 51 point SG to carry on the spectrum pretreatment, and selects 4 principal components modelling. In model 2, MSC was used for spectral pretreatment, and 8 principal components were selected. The model 3 uses the MSC +3 order 5 times 21 point SG to carry on the spectrum pretreatment, and selects 9 principal components modelling. It can be seen from table 1 that the classification results obtained with SG and 3 order 21 times 5 SG are the best.

Table 1. The classification effect of different pretreatment with PCA extraction

\begin{tabular}{cccc}
\hline Model & $\begin{array}{c}\text { Cumulative credibility } \\
(\%)\end{array}$ & $\begin{array}{c}\text { Modeling set accuracy } \\
(\%)\end{array}$ & $\begin{array}{c}\text { Test set accuracy } \\
(\%)\end{array}$ \\
\hline 1 & 91.8 & $96.7(58 / 60)$ & $90(18 / 20)$ \\
2 & 92.3 & $98.3(59 / 60)$ & $90(18 / 20)$ \\
3 & 97.3 & $100(60 / 60)$ & $100(20 / 20)$ \\
\hline
\end{tabular}

\section{CONCLUSIONS}

The spectral difference is because the infection degree root is not consistent. Due to the root of the roots of the infection after the decline of its roots to show the existence of the lag behind, as well as the stress of the protective effect of eggplant itself, resulting in a larger deviation of individual curves.

The leaves of Beijing 13 eggplant varieties with visible near infrared reflectance spectra data was analyzed in this paper. According to the characteristics of spectral curve, in different spectral bands the spectral data compressed to improve the efficiency of operation. the main research on MSC and SG pretreatment method and PCA principal component extraction method with the combination of effects on model for classification. Results show:The best classification process is to do the first MSC after SG smoothing pretreatment, after using PCA extracted as the main component of the SIMCA input variables for classification, and achieved a classification accuracy of $100 \%$. The results show that it is an effective method to classify the root knot nematodes and the degree of infection by using the visible and near infrared spectral characteristics of the eggplant leaves in the light of PCA and SIMCA.

\section{Ackknowledgements}

This work was supported by the National Key Research and Devement of China during the $13^{\text {th }}$ Five-Year Plan Period(No. 2017YFD0201607-2) and our research center Innovation team project(JNKST201619). Sincere thanks to Dr. Liu Ting for helping me complete the complex work of inoculating the eggplant with nematodes.

\section{REFERENCES}

1. Al-Hazmi A S, Al-Nadary S N. Interaction between Meloidogyne incognita and Rhizoctonia solani on green beans[J]. Saudi Journal of Biological Sciences, 2015, 27.

2. Abe N, Matsuzaki M, Wada K, et al. Influence of nematicide on the damage caused by Meloidogyne incognita (Tylenchida: Meloidogynidae) to sweet potato in sandy fields in 
northeastern Tokushima Prefecture[J]. Applied Entomology \& Zoology, 2015.

3. Anter A A, Amin A W, Ashoub A H, et al. Evaluation of inducers for tomato resistance against Meloidogyne incognita[J]. Pakistan Journal of Nematology, 2014.

4. Bello L Y, Chindo P S. Effects of some plant extracts on larval hatch of the root-knot nematode, Meloidogyne incognita[J]. Archives of Phytopathology \& Plant Protection, 2006, volume 39(4):253-257.

5. Claudius-Cole A O, Fawole B, Asiedu R, et al. Management of Meloidogyne incognita in yam-based cropping systems with cover crops[J]. Crop Protection, 2014, 63(5):97102.

6. Kumar A. Study on Pathogenicity Test of Meloidogyne incognita on Different Vegetable Crops[J]. Journal of Agricultural Science, 2015, 7(3):105-110.

7. Li D, Zhai M, Shi Q, et al. Comparison of host tropism and infection capacity of Me3 toxicities and non-toxicities of nematodes in south China [J]. Journal of plant pathology, 2018, 48(1):128-136.

8. Mukhtar T, Kayani M Z, Hussain M A. Response of selected cucumber cultivars to Meloidogyne incognita[J]. Crop Protection, 2013, 44(1):13 - 17.

9. Nishiyama H, Nakagami S, Todaka A, et al. Light-dependent green gall formation induced by Meloidogyne02incognita[J]. Nematology, 2014, 16.

10. Parsons J, Matthews W, Iorizzo M, et al. Meloidogyne incognita nematode resistance QTL in carrot[J]. Molecular Breeding, 2015, 35:1-11.

11. Pofu K M, Oelofse P W M D. Nematode resistance in bitter gourd to Meloidogyne incognita[J]. Acta Agriculturae Scandinavica, 2014, 65(1):1-5.

12. Siddiqui Z A, Shehzad M, Alam S. Interactions of Ralstonia solanacearum and Pectobacterium carotovorum with Meloidogyne incognita on potato[J]. Archives of Phytopathology \& Plant Protection, 2014, volume 47(4):449-455.

13. Xian Z H, Wang Y S, Hui-Jian A I, et al. The Synergistic Activity of Combination of Different Pesticides against Meloidogyne incognita(Root-Knot Nematode)[J]. Agrochemicals, 2014, 53(6):449-452.

14.Zijlstra C, Donkers-Venne DTHM, Farge e M. Identification of Meloidogyne incogni, M. javanica and M. arenaria using sequence characterised amplified region(SCAR)based PCR assays [J]. Nematology, 2000, 2: 847-853. 